\title{
Comprehensive assessment of the morbidity of renal mass biopsy: A population- based assessment of biopsy-related complications
}

\author{
Alaina Garbens ${ }^{1 *}$; Christopher J.D. Wallis ${ }^{1 *}$; Zachary Klaassen ${ }^{2}$; Refik Saskin ${ }^{3}$; \\ Lesley Plumptre ${ }^{4}$; Ronald Kodama ${ }^{1}$; Sender Herschorn ${ }^{1}$; Robert K. Nam ${ }^{1}$ \\ ${ }^{1}$ Division of Urology, Department of Surgery, Sunnybrook Health Sciences Centre, University of Toronto, \\ Toronto, ON, Canada; ${ }^{2}$ Section of Urology, Department of Surgery, Medical College of Georgia-Augusta \\ University, Augusta, GA, United States; ${ }^{3}$ Institute of Health Policy, Management \& Evaluation, University \\ of Toronto, Toronto, ON, Canada; ${ }^{4} \mathrm{ICES}$, Toronto, ON, Canada \\ *Equal contributors
}

Funding/Acknowledgment: Dr. Nam is supported by the Ajmera Family Chair in Urologic Oncology. This study made use of de-identified data from the ICES Data Repository, which is managed by the Institute for Clinical Evaluative Sciences with support from its funders and partners: Canada's Strategy for PatientOriented Research (SPOR), the Ontario SPOR Support Unit, the Canadian Institutes of Health Research and the Government of Ontario. The opinions, results and conclusions reported are those of the authors. No endorsement by ICES of any of its funders or partners is intended or should be inferred

Cite as: Can Urol Assoc J 2020 July 27; Epub ahead of print. http://dx.doi.org/10.5489/cuaj.6477

Published online July 27, 2020

$* * *$

\section{Abstract}

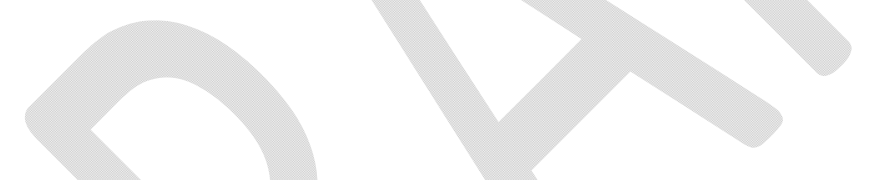

Introduction: We sought to assess seven-day and 30-day complications following renal mass biopsy (RMB), including mortality, hospitalizations, emergency department (ED) visits, and operative and non-operative complications and compare these to rates in population matched controls.

Methods: We performed a population-based, matched, retrospective cohort study of patients undergoing RMB following consultation with a urologist and axial imaging from 2003-2015 in Ontario, Canada. Data on seven-day and 30-day rates of mortality, operative and non operative complications after RMB were reported. The seven-day and 30-day rates of mortality, operative and non-operative interventions, hospitalizations, and ED visits were compared to matched controls using multivariable logistic regression. Results: Among 6840 patients who underwent RMB in the study period, $24(0.4 \%)$ and $159(2.3 \%)$ died within seven and 30 days, respectively, of their biopsy. Seven- and 30day operative intervention rates were $79(1.2 \%)$ and $236(3.4 \%)$, respectively. Sevenand 30-day non-operative intervention rates were 227 (3.3\%) and $529(7.7 \%)$ respectively. Thirty-day mortality (odds ratio [OR] 8.1; 95\% confidence interval [CI] 
5.1-13.0), hospitalizations (OR 12.6; 95\% CI 10.6-15.2), and ED visits (OR 3.8; 95\% CI 3.4-4.3) were more common among patients who underwent RMB than the matched controls ( $\mathrm{p}<0.001$ for each).

Conclusions: Patients undergoing RMB may have a small but non-negligible increased risk of mortality, hospital readmission, and ED visits compared to matched controls. However, limitations in the granularity of the dataset limits the strength of these conclusions. Further studies are needed to confirm our results. These risks should be discussed with patients for shared decision-making and considered in the risk/benefit tradeoff for the management of small renal masses.

\section{Introduction}

Increasing utilization of abdominal imaging has resulted in increased incidental diagnoses of small renal masses ${ }^{1}$. Approximately $80 \%$ of these masses are malignant ${ }^{2}$. Radiologic approaches to distinguish benign and malignant renal masses rely on characteristics including mass enhancement with intravenous contrast, size, location and growth parameters ${ }^{1}$.

Historically, the treatment for patients with small renal masses was surgical excision with partial or radical nephrectomy. A recent approach has been to perform a percutaneous renal mass biopsy (RMB) to establish a histologic diagnosis prior to definitive surgery. Currently, such an approach is controversial ${ }^{3,4}$ as the ability to acquire valuable diagnostic information that can risk stratify patients ${ }^{5}$ must be weighed against a non-diagnostic rate of approximately $15 \%$ and the risk of procedure-related complications ${ }^{6,7}$.

To date, data assessing outcomes of renal mass biopsy for small renal masses have been limited to single center studies ${ }^{5,6,8}$. Thus, we examined complication rates of renal mass biopsy in a large population within a single payer health care system, using mortality and morbidity endpoints.

\section{Methods}

We performed a population-based, retrospective cohort study of adults (aged $\geq 18$ years) in Ontario, Canada to examine the burden of complications associated with RMB. We used unique identifiers to link administrative databases of anonymized patient data held at the Institute for Clinical Evaluative Sciences (ICES) including the Ontario Health Insurance Plan (OHIP) database (physician billings, laboratories, and out-of-province providers) $)^{9}$; the Canadian Institute for Health Information (CIHI) Discharge Abstract Database (DAD; hospitalizations) $)^{10}$; the CIHI National Ambulatory Care Reporting System (NACRS; ambulatory and emergency room visits); the Ontario Cancer Registry (OCR; cancer diagnoses) ${ }^{11}$; the Ontario Drug Benefit (ODB; outpatient 
pharmaceuticals) ${ }^{12}$; and the Registered Persons Database (RPDB; demographic information $)^{13}$. The Sunnybrook Health Sciences Centre Research Ethics Board approved this study protocol.

\section{Patient population}

Using physician billings and hospital procedural records, we identified all patients undergoing a renal biopsy for presumed renal mass characterization (OHIP: Z601; CCI: 2.PC.71.HA, 2.PC.71.GR) between January 1, 2003 and March 31, 2015. As administrative data sources utilized do not distinguish between renal parenchymal biopsy and renal mass biopsy (RMB), we limited our cohort to those who had visited a urologist (specialty code 35) in the 6 months preceding or following biopsy and received an abdominal CT or MRI scan in the 12 months prior. Patients were excluded if they had received a RMB or renal cell cancer diagnosis prior to the index date.

\section{Study endpoints}

To characterise the complication burden of RMB, we examined 7-day and 30-day mortality, operative and non-operative intervention rates, hospitalizations and emergency department visits. We identified operative interventions using physician billings and hospital procedural records and characterized these as operative and non-operative (Supplemental Table 1). Operative interventions included total/radical nephrectomy, diagnostic laparotomy/laparoscopy, partial/total splenectomy, control of splenic bleeding, control of hepatic bleeding, control of bleeding from small and/or large intestine, bowel resection/repair and operative control of kidney bleeding. Non-operative interventions included renal angioembolization, percutaneous drain insertion, percutaneous nephrostomy and cystoscopic stent insertion.

Non-procedural complications included emergency room visits and hospital admissions within 30-days of RMB, identified using hospital records. We examined all such events and specifically examined those deemed urologically-related (urinary tract infection, pyelonephritis, abdominal pain/colic, renal colic, abdominal pain, hematuria, urinary extravasation and other urinary symptoms or disorders).

\section{Covariates}

To adjust for potential confounding, we collected data on patient characteristics. Patient level covariates included age, sex, comorbidity (Johns Hopkins University Aggregated Diagnosis Groups case mix system) ${ }^{14}$, geographic region of residence, year of biopsy, neighbourhood income quintile and blood thinner prescriptions up to 30 days prior to biopsy. We identified patients with chronic kidney disease on the basis of billing codes from nephrologists (ICD-10 diagnosis code N18.x). 


\section{Data analysis}

We descriptively characterised complications following RMB using counts with proportions for categorical variables and median with interquartile ranges for continuous variables. To assess the effect of renal biopsy on peri-procedural outcomes, we hard matched each patient undergoing biopsy 1:1 with a control drawn from the general population based on age, sex, comorbidity, geographic location, neighbourhood income quintile, and year. Finally, we performed logistic regression to assess the association between biopsy and complications and to identify predictors of complications following RMB. We performed a subset analyses among patients with no history of medical renal disease and those who may have metastatic disease on the basis of consultation with a medical oncologist 6 months prior to or after the date of RMB.

Model assumptions were verified, and no violations were identified. Statistical significance was set at $\mathrm{p}<0.05$ based on 2-tailed comparison. All analyses were performed using SAS Enterprise Guide 7.1 (SAS Institute Inc).

As the time interval between RMB and urologist consultation was arbitrarily set at 6 months, we assessed the effect of this assumption by repeating the analyses and varying this time interval to within 3 months and to within 1 year.

\section{Results}

During the study interval, 6,840 patients underwent renal biopsy and met all inclusion criteria for $\mathrm{RMB}$, including appropriate imaging and urologic consultation. Most patients were male, had multiple comorbidities and were aged 50 years or older (Table 1). Notably, 2286 (33.4\%) patients had a diagnosis of chronic kidney disease.

Of the 6,840 patients who underwent a RMB, 24 (0.4\%) and $159(2.3 \%)$ died within 7 and 30 days, respectively (Table 2). At 7 and 30 days following biopsy, complications requiring operative intervention occurred in $79(1.2 \%)$ and $236(3.4 \%)$ patients, respectively, and those requiring non-operative intervention occurred in 227 (3.3\%) and 529 (7.7\%; Table 2 and 3), respectively. 1,095 (16.0\%) patients visited the emergency department within 30 days of having a biopsy. Of those, $242(22.1 \%)$ were due to a urological cause (Table 3), with hematuria being the most common. 1,306 (19.1\%) patients were hospitalized within 30 days of biopsy, with 182 (13.9\%) being due to a urological cause. 7-day rates of intervention, hospitalization and ED visits are found in Table 2. When restricted to patients without chronic kidney disease $(n=4,554)$ and those without chronic kidney disease who also did not visit a medical oncologist ( $\mathrm{n}=$ 3,903), outcomes were comparable to the primary analysis (Tables 4 and 5).

We matched 6,769 patients who underwent RMB during the study period to 6,769 controls (Table 1). Patients who underwent RMB had significantly higher rates mortality at 7 -days $(0.4 \%$ vs. less than $0.07 \% ; \mathrm{p}=0.0007)$ and 30 -days $(2.3 \%$ vs. $0.3 \%$; number needed to treat to harm (NNTH) 49, 95\% CI 41-59) following RMB. Additionally, rates of emergency department visits at 7-days (5.6\% vs. 1.4\%; NNTH $24,95 \%$ CI $21-28$ ) and 
30-days ( $15.9 \%$ vs. $4.7 \%$; NNTH 9, 95\% CI 8-10) following index were higher among those who underwent RMB. Finally, rates of hospitalization were higher at 7-days (10\% vs. $0.5 \%$; NNTH $11,95 \%$ CI $10-11)$ and 30 -day (19.1\% vs. $1.8 \%$, OR $12.6,95 \%$ CI $10.4-$ $15.2, \mathrm{p}<0.0001)$ and 30 -days $(19.1 \%$ vs. $1.8 \%$; NNTH $6,95 \%$ CI $5-6)$ compared to controls (Table 2). Use of blood thinners was not a predictor of complications, admissions or emergency department visits on multivariable analysis (OR 1.0, 95\% CI $0.76-1.31, \mathrm{p}=0.99$ ).

Across analyses, sensitivity analyses varying the exposure window did not significantly change the study results (data not shown).

\section{Discussion}

Utilizing a large, population-based cohort, we identified low but not insignificant rates of mortality (2.3\%) and complications (up to $10 \%$ ) within 30 -days of RMB. These results were robust in subgroup analyses excluding patients with medical renal disease and suspicion of metastatic disease. Rates of hospitalization and ED visits were higher among patients undergoing RMB than population matched controls. Due to the limitations of these data, we are unable to directly attribute either the mortality or morbidity experienced by these patients to their RMB. Indeed, it is likely that physicians opt to perform RMB specifically in patients who are at increased risk of peri-procedural morbidity. However, these data highlight the potential outcomes a patient who is considering RMB should be aware of prior to undertaking the procedure.

To our knowledge, this is the first population-level report of complications related to RMB. Single institution series have demonstrated a low rate of complications $(<10 \%)$, with a preponderance of Clavien-Dindo Grade 1 peri-renal hematomas incidentally detected on postprocedural imaging ${ }^{5,6}$. In centers in which asymptomatic perinephric hematomas are not routinely sought, reported complication rates are event lower $(<4 \%)^{15}$. Rare cases of bleeding requiring embolization ${ }^{5,15,16}$ or hospitalization for bladder irrigation ${ }^{6}$, Clavien-Dindo Grade 3 events, have been reported. Systematic reviews of such series have corroborated these findings and concluded that major complications are rare $^{17,18}$. However, as these studies were limited to academic institutions, the generalizability of these findings are unclear ${ }^{19}:$ in contrast, the present study provides generalizable results which may be reliably extrapolated to general medical practice.

Current guidelines for the management of small renal masses neither advocate nor oppose the use of $\mathrm{RMB}^{4,20}$. Opponents to widespread use of RMB have focused on concerns regarding accuracy and safety ${ }^{19}$. While we did not assess the accuracy of RMB in this analysis, diagnostic success rates in excess of $90 \%$ have been documented in both single institutional series ${ }^{5}$ and systematic reviews ${ }^{21}$. Concerns have been raised that RMB may represent an unnecessary test which may be associated with increased patient anxiety, a risk of unnecessary harm, waste of valuable healthcare resources, and potentially even delays in definitive treatment ${ }^{22}$. However, we recently found that 
patients who underwent a RMB prior to radical or partial nephrectomy had significantly lower rates of benign disease on surgical pathology ${ }^{23}$. Therefore, routine use of RMB may reduce rates of renal surgery for benign disease. This may avoid significant morbidity as well as cost. Further, a recent review of cost-effectiveness studies assessing the management of small renal masses demonstrated that a biopsy-based strategy (with possible subsequent intervention) dominated immediate surgical intervention, yielding improved outcomes at lower costs ${ }^{24}$.

Strengths of this analysis include its generalizable nature and the robust identification of both exposure and outcomes. This study was performed in Ontario, Canada, a jurisdiction in which all relevant health services are available free of cost and are systematically tracked in administrative databases. Thus, both exposure and outcome are accurately and comprehensively captured. Further, all physician interactions, procedures, emergency room visits, and hospitalizations are captured, regardless of where in the province they occurred, thus eliminating ascertainment bias associated with institutional studies.

However, a number of limitations are present. First, the definition of nonprocedural complications was restricted to hospitalizations and emergency room visits, excluding outpatient office physician interactions. These are likely to capture the vast majority of significant complications and the validity of these diagnoses has been well established in Ontario ${ }^{10}$. Second, operative and non-operative procedures were used as surrogates for actual complications without an ability to ascertain the indication for each intervention. This is perhaps most relevant for the outcome of nephrectomy as a complication following renal mass biopsy. While nephrectomy may be necessitated in rare circumstances to manage bleeding, it may also represent definitive management of a previously biopsied lesion. However, given surgical wait times in Ontario, it is uncommon for patients with genitourinary malignancies to undergo surgery within 7 or 30 days of the decision to operate, a time that will be delayed from the date of RMB due to the time required for pathologic examination of the biopsy specimen. However, misattribution of these cases would lead to an overestimate of complications rates. Thus, true rates may be lower than estimated in this analysis. Third, we are unable to directly attribute any of the outcomes to biopsy. In particular, for mortality, we are unable to ascertain cause of death. However, compared a matched cohort from the general population, mortality rates within 30-days of biopsy were significantly higher. Fourth, due to limitations in the administrative data sources utilized, we lack details regarding the renal mass including size, location, and complexity and regarding specific pathologic outcomes. As stated earlier, while we attempted to exclude patients who underwent renal biopsy for medical renal disease, as the same procedure code is used for both RMB and renal biopsy. As a result, we could not definitively exclude renal biopsy from our population. Thus, we performed sensitivity analyses to assess the effect of this on study 
conclusions. Finally, due to the administrative nature of our data, we were unable to collect relevant patient reported outcomes.

\section{Conclusions}

In a large, population-based cohort, renal mass biopsy may be associated with a low, but non-negligible, rate of mortality, operative and non-operative complications, hospitalization and emergency department visits. However, due to the limitations of our population-based data, further studies are needed to confirm our results. These events, along with the potential benefits of renal mass biopsy, should be considered in shared decision making before proceeding to biopsy. 


\section{References}

1. Gill, I. S., Aron, M., Gervais, D. A. et al.: Clinical practice. Small renal mass. N Engl J Med, 362: 624, 2010

2. Frank, I., Blute, M. L., Cheville, J. C. et al.: Solid renal tumors: an analysis of pathological features related to tumor size. J Urol, 170: 2217, 2003

3. Patel, H. D., Johnson, M. H., Pierorazio, P. M. et al.: Diagnostic Accuracy and Risks of Biopsy in the Diagnosis of a Renal Mass Suspicious for Localized Renal Cell Carcinoma: Systematic Review of the Literature. The Journal of Urology, 195: 1340, 2016

4. Campbell, S., Uzzo, R. G., Allaf, M. E. et al.: Renal Mass and Localized Renal Cancer: AUA Guideline. The Journal of Urology, 2017

5. Richard, P. O., Jewett, M. A. S., Bhatt, J. R. et al.: Renal Tumor Biopsy for Small Renal Masses: A Single-center 13-year Experience. European Urology, 68: 1007, 2015

6. Leveridge, M. J., Finelli, A., Kachura, J. R. et al.: Outcomes of small renal mass needle core biopsy, nondiagnostic percutaneous biopsy, and the role of repeat biopsy. Eur Urol, 60: 578, 2011

7. Prince, J., Bultman, E., Hinshaw, L. et al.: Patient and Tumor Characteristics can Predict Nondiagnostic Renal Mass Biopsy Findings. The Journal of Urology, 193: 1899,2015

8. Halverson, S. J., Kunju, L. P., Bhalla, R. et al.: Accuracy of determining small renal mass management with risk stratified biopsies: confirmation by final pathology. J Urol, 189: 441, 2013

9. Williams, J. I., Young, W.: A summary of studies on the quality of health care administrative databases in Canada. In: Patterns of Health Care in Ontario, Canada: The ICES Practice Atlas. Edited by V. Goel, J. Williams, G. Anderson et al. Ottawa, Ontario, Canada: Canadian Medical Association, pp. 339-345, 1996

10. Juurlink, D. N., Preyra, C., Croxford, R. et al.: Canadian Institute for Health Information Discharge Abstract Database: A validation study. Toronto, Ontario, Canada: Institute for Clinical Evaluation Sciences, 2006

11. Robles, S. C., Marrett, L. D., Clarke, E. A. et al.: An application of capturerecapture methods to the estimation of completeness of cancer registration. J Clin Epidemiol, 41: 495, 1988

12. Levy, A. R., O'Brien, B. J., Sellors, C. et al.: Coding accuracy of administrative drug claims in the Ontario Drug Benefit database. Can J Clin Pharmacol, 10: 67, 2003

13. Iron, K., Zagorski, B. M., Sykora, K. et al.: Living and Dying in Ontario: An Opportunity for Improved Health Information. Toronto, ON: ICES Investigative Report, 2008

14. Health., J. H. H. B. S. o. P.: The Johns Hopkins ACG Case-Mix System Reference Manual Version 9.0. Baltimore, MD: The Johns Hopskins University, 2009 
15. Graumann, O., Rasmussen, L. R., Loft, M. et al.: Do we need a post-biopsy observation period following ultrasound guided biopsies of renal masses? Scandinavian Journal of Urology, 51 (220): 49, 2017

16. Levi, J., Kimche, D., Lerner, M. A.: Early angiography in the management of post-renal biopsy hematuria. Journal of Urology, 119: 410, 1978

17. Volpe, A., Kachura, J. R., Geddie, W. R. et al.: Techniques, safety and accuracy of sampling of renal tumors by fine needle aspiration and core biopsy. J Urol, 178: 379, 2007

18. Volpe, A., Finelli, A., Gill, I. S. et al.: Rationale for percutaneous biopsy and histologic characterisation of renal tumours. Eur Urol, 62: 491, 2012

19. Capitanio, U., Volpe, A.: Renal Tumor Biopsy: More Dogma Belied. Eur Urol, 68: 1014,2015

20. Jewett, M. A., Rendon, R., Lacombe, L. et al.: Canadian guidelines for the management of small renal masses (SRM). Can Urol Assoc J, 9: 160, 2015

21. Lane, B. R., Samplaski, M. K., Herts, B. R. et al.: Renal mass biopsy--a renaissance? J Urol, 179: 20, 2008

22. Khorasani, R., Hentel, K., Darer, J. et al.: Ten commandments for effective clinical decision support for imaging: enabling evidence-based practice to improve quality and reduce waste. AJR Am J Roentgenol, 203: 945, 2014

23. Wallis, C. J. D., Garbens, A., Klaassen, Z. et al.: Effect of renal mass biopsy on subsequent nephrectomy outcomes: a population-based assessment. Eur Urol, (epublished October 2), 2019

24. Wang, Y., Chen, Y. W., Leow, J. J. et al.: Cost-effectiveness of Management Options for Small Renal Mass: A Systematic Review. Am J Clin Oncol, 39: 484, 2016

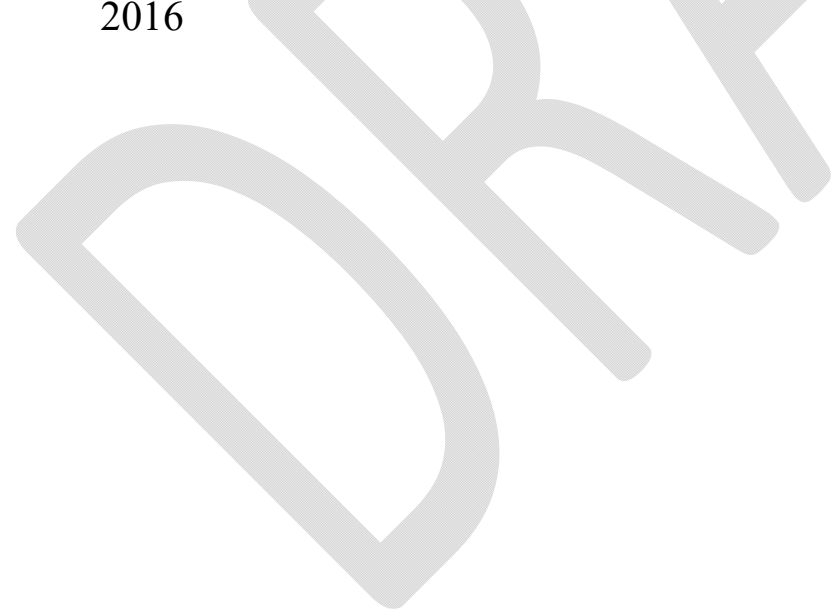


Figures and Tables

\begin{tabular}{|c|c|c|}
\hline \multicolumn{3}{|c|}{$\begin{array}{l}\text { Table 1. Baseline demographic characteristics of patients putatively } \\
\text { undergoing renal mass biopsy between January } 1,2003 \text { and March } \\
\text { 31, 2015, and matched controls from the general population }\end{array}$} \\
\hline Baseline characteristics & RMB & $\begin{array}{l}\text { Matched } \\
\text { controls }\end{array}$ \\
\hline Number & 6769 & 6769 \\
\hline Median (IQR) & $64(54-73)$ & $64(54-73)$ \\
\hline $18-39$ & $472(7.0 \%)$ & $472(7.0 \%)$ \\
\hline $40-49$ & $712(10.5 \%)$ & $712(10.5 \%)$ \\
\hline $50-59$ & $1336(19.7 \%)$ & $1336(19.7 \%)$ \\
\hline $60-69$ & $1857(27.4 \%)$ & $1857(27.4 \%)$ \\
\hline $70+$ & $2392(35.3 \%)$ & $2392(35.3 \%)$ \\
\hline \multicolumn{3}{|l|}{ Sex, n (\%) } \\
\hline Female & $2504(37.0 \%)$ & $2504(37.0 \%)$ \\
\hline Male & $4265(63.0 \%)$ & $4265(63.0 \%)$ \\
\hline \multicolumn{3}{|l|}{ Income quintile, n (\%) } \\
\hline 1 - Lowest & $1314(19.4 \%)$ & $1314(19.4 \%)$ \\
\hline 2 & $1396(20.6 \%)$ & $1396(20.6 \%)$ \\
\hline 3 & $1334(19.7 \%)$ & $1334(19.7 \%)$ \\
\hline 4 & $1345(19.9 \%)$ & $1345(19.9 \%)$ \\
\hline 5 - Highest & $1380(20.4 \%)$ & $1380(20.4 \%)$ \\
\hline \multicolumn{3}{|c|}{ Aggregated Diagnosis Group (ADG) categories } \\
\hline $1-4$ & $334(4.9 \%)$ & $334(4.9 \%)$ \\
\hline $5-9$ & $2885(42.6 \%)$ & $2885(42.6 \%)$ \\
\hline $10+$ & $3550(52.4 \%)$ & $3550(52.4 \%)$ \\
\hline \multicolumn{3}{|l|}{ Year } \\
\hline $2003-2005$ & $894(13.2 \%)$ & $894(13.2 \%)$ \\
\hline $2006-2008$ & $1146(16.9 \%)$ & $1146(16.9 \%)$ \\
\hline $2009-2011$ & $1786(26.4 \%)$ & $1786(26.4 \%)$ \\
\hline $2012-2015$ & $2943(43.5 \%)$ & $2943(43.5 \%)$ \\
\hline
\end{tabular}

IQR: interquartile range; RMB: renal mass biopsy. 


\begin{tabular}{|c|c|c|c|c|c|c|c|c|}
\hline \multirow[t]{2}{*}{ Outcome } & \multicolumn{4}{|c|}{ 7-day outcomes } & \multicolumn{4}{|c|}{ 30-day outcomes } \\
\hline & $\begin{array}{l}\text { RMB, } \\
\text { n (\%) }\end{array}$ & $\begin{array}{c}\text { Controls, } \\
\text { n (\%) }\end{array}$ & $\begin{array}{c}\text { OR } \\
(95 \% \mathrm{CI})\end{array}$ & $\begin{array}{c}\text { Absolute risk } \\
\text { difference, \% } \\
\text { (95\% CI) }\end{array}$ & $\begin{array}{l}\text { RMB, } \\
\text { n (\%) }\end{array}$ & $\begin{array}{c}\text { Controls, } \\
\text { n }(\%)\end{array}$ & $\begin{array}{c}\text { OR } \\
(95 \% \text { CI })\end{array}$ & $\begin{array}{c}\text { Absolute } \\
\text { risk } \\
\text { difference, } \\
\%(95 \% \mathrm{CI})\end{array}$ \\
\hline Mortality & $\begin{array}{c}24 \\
(0.4)\end{array}$ & $1-5^{* *}$ & \multicolumn{2}{|c|}{ Unable to report $^{* *}$} & $159(2.3)$ & $20(0.3)$ & $\begin{array}{c}8.1 \\
(5.1-13.0)\end{array}$ & $\begin{array}{c}2.1 \\
(1.7-2.5)\end{array}$ \\
\hline Hospitalizations & $\begin{array}{l}683 \\
(10)\end{array}$ & $36(0.5)$ & $\begin{array}{c}20.8(14.8- \\
29.1) \\
\end{array}$ & $9.5(8.7-10.2)$ & $\begin{array}{l}1,306 \\
(19.1) \\
\end{array}$ & $124(1.8)$ & $\begin{array}{c}12.6 \\
(10.6-15.2) \\
\end{array}$ & $\begin{array}{c}17.2 \\
(16.2-18.2) \\
\end{array}$ \\
\hline Emergency visits & $\begin{array}{c}383 \\
(5.6)\end{array}$ & $96(1.4)$ & $4.2(3.3-5.2)$ & $4.2(3.6-4.8)$ & $\begin{array}{l}1,095 \\
(15.9)\end{array}$ & $320(4.7)$ & $\begin{array}{c}3.8 \\
(3.4-4.3)\end{array}$ & $\begin{array}{c}11.2 \\
(10.2-12.2)\end{array}$ \\
\hline
\end{tabular}

${ }^{*}$ Controls were hard matched for age, sex, comorbidity (ADG), geographic location, neighbourhood income quintile, and year of procedure. ${ }^{* *}$ Values suppressed due to small cells due to administrative policy that individual counts less than 6 cannot be reported. As a result, OR and absolute risk difference cannot be reported due to the potential to derive these small values. CI: confidence interval; OR: odds ratio; RMB: renal mass biopsy. 


\begin{tabular}{|c|c|c|}
\hline \multirow[t]{2}{*}{ Outcome } & \multicolumn{2}{|c|}{ Renal mass biopsy } \\
\hline & 7-day, n (\%) & 30-day, n (\%) \\
\hline Operative interventions (total) & $79(1.2)$ & $236(3.4)$ \\
\hline Radical nephrectomy & $39(0.6)$ & $151(2.2)$ \\
\hline Diagnostic laparotomy or laparoscopy & $27(0.4)$ & $43(0.6)$ \\
\hline Bowel resection/repair & $13(0.2)$ & $42(0.6)$ \\
\hline Non-operative interventions (total) & $227(3.3)$ & $529(7.7)$ \\
\hline Renal angioembolization & $44(0.6)$ & $61(0.9)$ \\
\hline Percutaneous drain insertion & $161(2.4)$ & $419(6.1)$ \\
\hline Cystoscopic stent insertion & $22(0.3)$ & $49(0.7)$ \\
\hline Hospitalizations & & \\
\hline Any & $683(10)$ & $1,306(19.1)$ \\
\hline Urologically related ${ }^{*}$ & $88(1.3)$ & $182(2.7)$ \\
\hline Emergency visits & +7 & P \\
\hline Any & $383(5.6)$ & $1,095(16.0)$ \\
\hline Urologically related* & $94(1.4)$ & $242(3.5)$ \\
\hline
\end{tabular}

*Includes urinary tract infection, pyelonephritis, renal colic, abdominal pain, hematuria, urinary extravasation, and other urinary symptoms or disorders. 


\begin{tabular}{|c|c|c|}
\hline Outcome & 7-day, n (\%) & 30-day, n (\%) \\
\hline Mortality & $25(1.8 \%)$ & $94(2.1 \%)$ \\
\hline Operative interventions (total) & $47(1.0)$ & $164(3.6)$ \\
\hline Radical nephrectomy & $25(0.5)$ & $117(2.6)$ \\
\hline $\begin{array}{l}\text { Diagnostic laparotomy or } \\
\text { laparoscopy }\end{array}$ & $14(0.3)$ & $19(0.4)$ \\
\hline Bowel resection/repair & $8(0.2)$ & $28(0.6)$ \\
\hline Non-operative interventions (total) & $95(2.1)$ & $232(5.1)$ \\
\hline Renal angioembolization & $27(0.6)$ & $36(0.8)$ \\
\hline Percutaneous drain insertion & $53(1.2)$ & $163(3.6)$ \\
\hline Cystoscopic stent insertion & $15(0.3)$ & $33(0.7)$ \\
\hline \multicolumn{3}{|l|}{ Hospitalizations } \\
\hline Any & $366(8.0)$ & $722(15.9)$ \\
\hline Urologically related $^{*}$ & $61(1.3)$ & $96(2.1)$ \\
\hline \multicolumn{3}{|l|}{ Emergency visits } \\
\hline Any & $250(5.5)$ & $621(13.6)$ \\
\hline Urologically related* & $62(1.4)$ & $143(3.1)$ \\
\hline
\end{tabular}

*Includes urinary tract infection, pyelonephritis, renal colic, abdominal pain, hematuria, urinary extravasation, and other urinary symptoms or disorders. CT: computed tomography; MRI: magnetic resonance imaging. 


\begin{tabular}{|c|c|c|}
\hline \multicolumn{3}{|c|}{$\begin{array}{l}\text { Table 5. Short-term post-procedural complications of patients without } \\
\text { chronic renal disease who underwent renal mass biopsy }(n=3903) \text {, saw a } \\
\text { urologist, did not see a medical oncologist, and had a CT or MRI scan within } \\
12 \text { months }\end{array}$} \\
\hline Outcome & 7-day, n (\%) & 30-day, n (\%) \\
\hline Mortality & $9-13^{* *}$ & $78(2.0 \%)$ \\
\hline \multicolumn{3}{|l|}{ Operative interventions } \\
\hline Radical nephrectomy & $20-24^{* *}$ & $94(2.4 \%)$ \\
\hline $\begin{array}{l}\text { Diagnostic laparotomy or } \\
\text { laparoscopy }\end{array}$ & $9-13^{* *}$ & $14-18^{* *}$ \\
\hline Bowel resection/repair & $3-7^{* *}$ & $22-27^{* *}$ \\
\hline \multicolumn{3}{|c|}{ Non-operative interventions (total) } \\
\hline Renal angioembolization & $22-26^{* *}$ & $31-35^{* *}$ \\
\hline Percutaneous drain insertion & $40(1.0 \%)$ & $125(3.2 \%)$ \\
\hline Cystoscopic stent insertion & $10-14^{* *}$ & $23(0.6 \%)$ \\
\hline \multicolumn{3}{|l|}{ Hospitalizations } \\
\hline Any & $323(8.3 \%)$ & $596(15.3 \%)$ \\
\hline Urologically related $^{*}$ & $55(1.4 \%)$ & $82(2.1 \%)$ \\
\hline \multicolumn{3}{|l|}{ Emergency visits } \\
\hline Any & $195(5.0 \%)$ & $490(12.6 \%)$ \\
\hline Urologically related $* \varphi$ & $52(1.3 \%)$ & $117(3.0 \%)$ \\
\hline
\end{tabular}

*Includes urinary tract infection, pyelonephritis, renal colic, abdominal pain, hematuria, urinary extravasation, and other urinary symptoms or disorders. ${ }^{* *}$ Cells numbers suppressed due to small numbers. CT: computed tomography; MRI: magnetic resonance imaging. 


\begin{tabular}{|c|c|}
\hline \multicolumn{2}{|c|}{ Supplementary Table 1. Procedural codes and diagnostic codes } \\
\hline Description & Code used \\
\hline Percutaneous renal mass biopsy & OHIP: Z601; CCI: 2.PC.71.HA \\
\hline \multicolumn{2}{|l|}{ Treatments } \\
\hline Partial nephrectomy & OHIP S411, S423; CCI 1.PC.87.^^ \\
\hline Radical nephrectomy & $\begin{array}{l}\text { OHIP S413, S415, S416; CCI } \\
1 . P C .89 .^{\wedge}, 1 . P C .91 .^{\wedge}\end{array}$ \\
\hline Percutaneous cryoablation/RFA & $\begin{array}{l}\text { OHIP J069; CCI 1.PC.59.HA-X7, } \\
\text { 1.PC.59.HA-AW, 1.PC.HA-CG }\end{array}$ \\
\hline Open cryoablation/RFA & $\begin{array}{l}\text { CCI 1.PC.59.LA-X7, 1.PC.59.LA- } \\
\text { AW }\end{array}$ \\
\hline Laparoscopic cryoablation/RFA & $\begin{array}{l}\text { OHIP S400; CCI 1.PC.59.BA-X7, } \\
\text { 1.PC.59.BA-AW }\end{array}$ \\
\hline Radiotherapy & 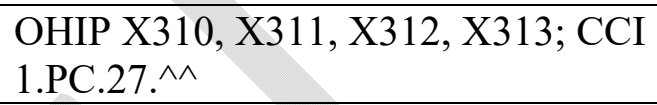 \\
\hline \multicolumn{2}{|l|}{ Operative complications } \\
\hline \multicolumn{2}{|l|}{ Diagnostic laparotomy/laparoscopy } \\
\hline Partial/total splenectomy & 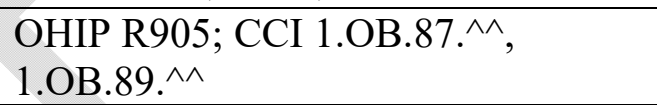 \\
\hline Control of splenic bleeding & CCI 1.OB.13.^^ \\
\hline Control of hepatic bleeding & CCI 1.OA.13.^^ \\
\hline Control of bleeding, small and large intestine & $1 . N P .13 .^{\wedge \wedge}$ \\
\hline Repair of small bowel & OHIP S184; CCI 1.NK.80.^^ \\
\hline Partial excision of small bowel & OHIP S164, S165; CCI 1.NK.87.^^ \\
\hline Repair of large bowel & CCI 1.NM.80.^^ \\
\hline Partial excision of large bowel & 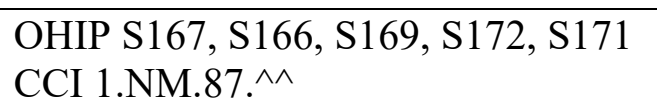 \\
\hline Operative control of kidney bleeding & CCI 1.PC.13.LA, 1.PC.80.^^ \\
\hline \multicolumn{2}{|l|}{ Non-operative complications } \\
\hline Renal angioembolization & $\begin{array}{l}\text { OHIP J040; CCI 1.PC.13.GQ-C2, } \\
\text { 1.PC.13.GQ-GE, 1.PC.13.GQ-W0 }\end{array}$ \\
\hline $\begin{array}{l}\text { Percutaneous drainage of abdominal } \\
\text { cavity/retroperitoneum }\end{array}$ & $\begin{array}{l}\text { CCI 1.OT.52.HA, 1.OT.52.HA-TS, } \\
\text { 1.OT.52.HH-D1, 1.OT.52.HH-D2, } \\
\text { 1.OT.52.HH-D3 }\end{array}$ \\
\hline Percutaneous drainage of soft tissue & CCI 1.SZ.52.HA, 1.SZ.52.HA-TS \\
\hline Percutaneous abdominal abscess drainage & OHIP Z594 \\
\hline Drainage of kidney abscess or perinephric abscess & OHIP S401, S402 \\
\hline Ultrasound guided biopsy, aspiration, or drainage & OHIP J149 \\
\hline Drainage of subfascial abscess & OHIP Z410 \\
\hline Percutaneous nephrostomy & OHIP J046; CCI 1.52.PC.HA \\
\hline Cystoscopic stent insertion & OHIP E818; CCI 1.PE.50.BA-BJ \\
\hline
\end{tabular}




\begin{tabular}{|l|l|}
\hline \multicolumn{2}{|l|}{ Hospital admission with following diagnoses } \\
\hline Urinary tract infection & ICD-10 N10, N30, N39.0 \\
\hline Pyelonephritis & ICD-10 N10.x \\
\hline Abdo pain: colic & ICD-10 R10.83 \\
\hline Unspecified renal colic & ICD-10 N23.x \\
\hline Abdominal pain & ICD-10 R10.1, R10.2, R10.3, R10.81, \\
& R10.84, R10.9 \\
\hline Hematuria & ICD-10 R31.x \\
\hline Urinary extravasation & ICD-10 R39.0 \\
\hline Other urinary symptoms or disorders & ICD-10 N39.9, R39.9 \\
\hline
\end{tabular}

CCI: Canadian Classification of Health Interventions; OHIP: Ontario Health Insurance Plan; RFA: radiofrequency ablation. Both ${ }^{\wedge}$ and $\mathrm{x}$ are placeholders that represent any number. 